\title{
An Adaptive QoS Mechanism for Multimedia Applications over Next Generation Vehicular Network
}

\author{
Jiann-Liang Chen \\ Dept. of Electrical Engineering, \\ National Taiwan University of Science \\ \& Technology, \\ Taipei, Taiwan. \\ lchen@mail.ntust.edu.tw
}

\author{
Yi-Wei Ma, Yueh-Min Huang \\ Dept. of Engineering Science, \\ National Cheng Kung University, \\ Tainan, Taiwan. \\ n9897106@mail.ncku.edu.tw \\ huang@mail.ncku.edu.tw
}

\author{
Qing-Tai Yang \\ Institute of Electronic Engineering, \\ National Dong Hwa University, \\ Hualien, Taiwan.
}

\begin{abstract}
This study develops a novel IEEE 802.21 MIH (Media Independent Handover) mechanism for next generation vehicular multimedia network. An adaptive QoS management mechanism is also proposed. By obtaining received signal strength parameters, the proposed MIH framework can determine the best available network. The adaptive QoS mechanism substantially improves the performance of real-time multimedia applications. The simulation results show that average handover time is slower than both UMTS and WiMAX when the MIH mechanism is used in vehicular network. However, the simulation results confirm that using the IEEE 802.21 MIH mechanism can increase overall throughput. Increased throughput is satisfactory compensation for increased handover time.
\end{abstract}

Keywords-Vehicular Network; Next Generation Network; Media-Independent Handover; Quality of Service.

\section{INTRODUCTION}

Future vehicular networks may require mobile devices to cross over different servicing locations. Maintaining network services requires seamless integration of different networks. An in-vehicle user roaming between different wireless communications technologies requires efficient handover between different networks and continuous connections of applications in the different networks. However, advanced multimedia services require increasing network bandwidth and improved quality. To satisfy the high quality requirements of heterogeneous vehicular network users, an effective QoS mechanism is needed.

The rapid deployment of the wireless networks now enables MN (Mobile Node) to provide continuous internet access when it is moving. An MN located in an access network with weak strength must optimize network quality by performing handover conversion. In the past, MN could only provide handover in homogeneous networks. This technique does not enable the balance of network load into different networks. Figure 1 shows how MN can access different networks in a next-generation network architecture that includes $3 \mathrm{G}$ and WiMAX access technologies. When $\mathrm{MN}$ moves across different wireless networks, handover procedure automatically performs network optimization [1].

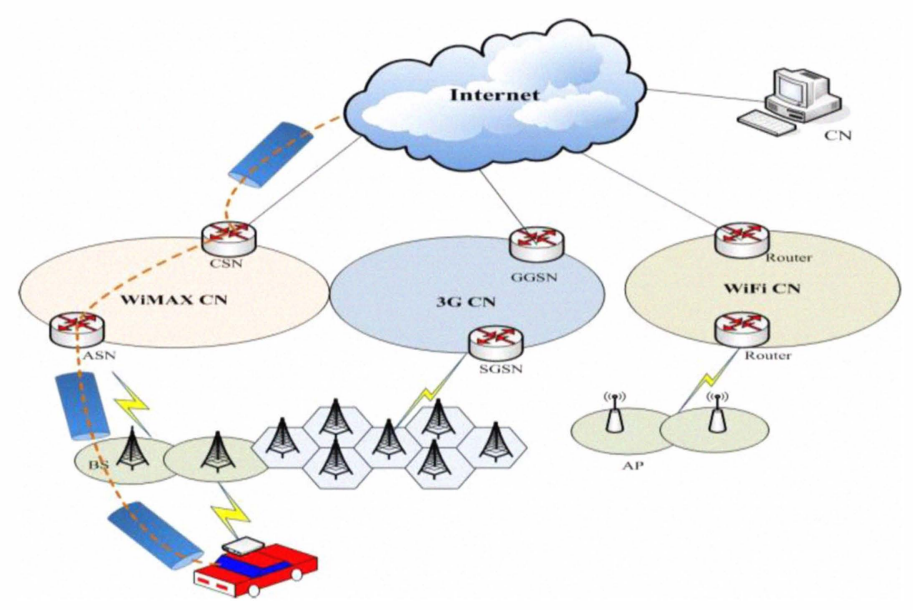

Figure 1. Vehicular Network

This study designs MIH functions and then embeds them into OSI layers to support multimedia applications over heterogeneous networks [2]. The MIH layer supports handover processing for in-vehicle users. This study also enhances the application layer by developing a network management module that contains an adaptive QoS mechanism needed for highquality multimedia services.

\section{BACKGROUND KNOWLEDGE}

Overlapping networks provide alternative wireless links such as WLAN and 3G. Mobile users are connected to the best network according to predefined priorities. Dynamic network accesses technologies are still needed to improve the distribution of network resources standards have been developed to support dynamically roaming users in heterogeneous network environments.

\section{A. IETF Network Mobility}

The SIP is a flexible, multi-purpose signaling protocol for session-based communications in IP networks. Since the SIP only handles the session management phase, different communication applications can be used after the session has 
been established. Two essential logical elements in the SIP architecture are registrar and proxy servers. Registrars are the SIP entities in which SIP users register their contact information after connecting to a network.

Regarding next-generation value-added network services, system operators must re-integrate the inherent network with the mobile network, and create a new network architecture in which the traditional line replaces the IP network as the core network and in which the center and the fixed network, wireless network and GSM/GPRS/3G network are integrated.? The new network infrastructure is the IMS [3].

\section{B. IEEE MIH Architecture}

To integrate heterogeneous wireless network, the IEEE 802.21 standard defined by IEEE organizations is the MIH Mechanism. Its main purpose is to develop a handover mechanism to provide a common communication interface to the IEEE series of each system so that the interface between the two different systems can provide effective communication. The MIH mechanism also includes the cross-integration scheme of heterogeneous network interface to provide a qualitative abnormality of the hands network optimization the MIH will import the existing 3G system mainly through cellular network integration [4-6] .

The IEEE 802.21 instead of the definition of MIH intermediary protocol layer to layer 2 . The network information communicates with the layer 3 protocol and the upper layers so that the abnormal mass MN network environment can optimize handover. Figure 2 shows the MIH architecture. The Link layer is between MIH Function and the Network layer. Upper layers can determine the network conditions in the lower layer through the MIH Function.

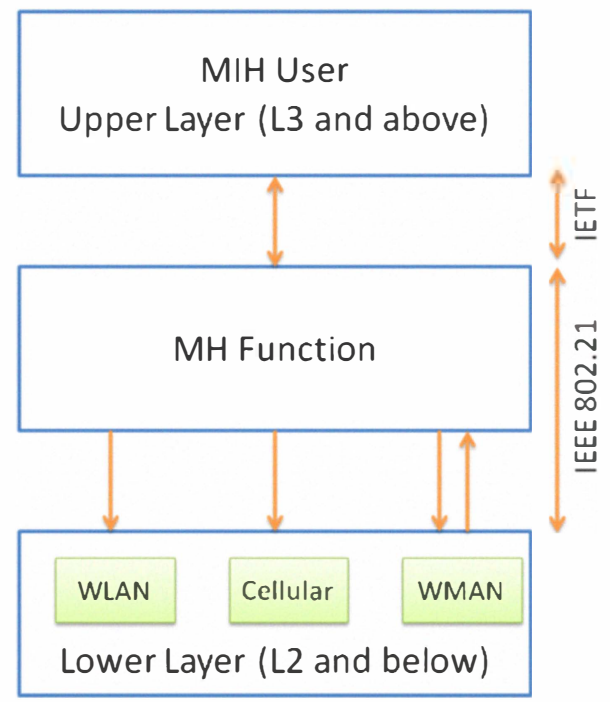

Figure 2. MIH Protocol Stack

For the purpose of MN, handover in the MNs gradually moves away from the original BS, and the communication signal weakens. MN needs at this time switch to other hearing a good strong BS. As for the stationary users, handover demand occurred in the user wishes to switch channels forced to improve the transmission performance.
The MN is connected with the original link and the layer 2 network information is sensed, and after the MIH function confirms that the signal for less than threshold and upper layer of the threshold issue of event notification, when received by upper layer of the 'Link Going Down' event notification. Upper layer MIH user can send a lower layer requirement of changing hands. When changing hands on the trigger event, the relative order should be issued to collect the media information network message and the MIH Function provides all mechanisms needed for the three services. The three services are Event Service, Command Service and Information Service.

\section{Proposed System ARCHITECTURE}

This study proposes a new network architecture that combines SIP scheme and MIH mechanism. Management mechanisms are implemented in application layer, and MIHF provides the handover function in the upper layers. The mechanism provides the MIHF network environment parameters and handover mechanisms needed for effective MN handover in heterogeneous networks.

Figure 3 shows the SIP Server, MIIS Server, CN and three different network environments, including WiFi and 3G networks. The SIP Server provides a register and a proxy to deal with the MN mobility function. The study uses SIP to support terminal mobility, and it remains connected when the terminal equipment is moved between different sub-networks.

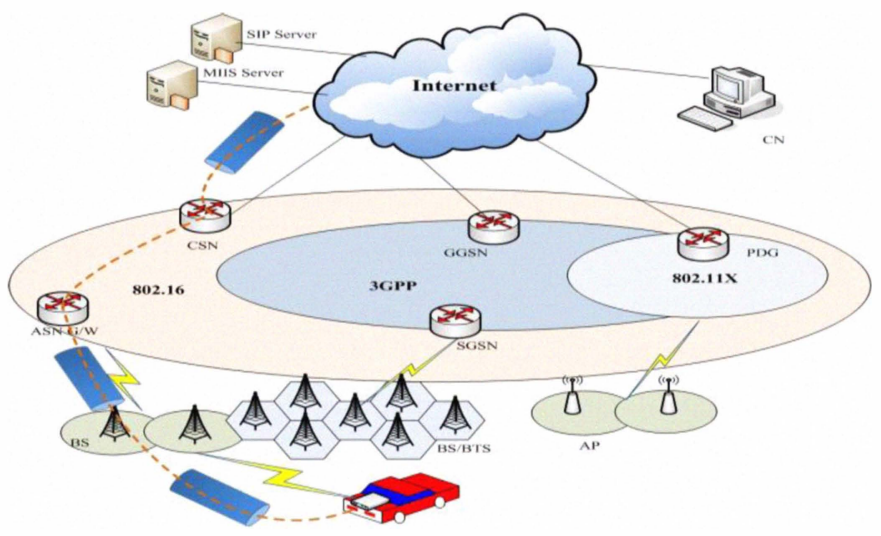

Figure 3. Proposed Vehicular Network Architecture

The MIIS server on the core network stores available network information to help $\mathrm{MN}$ perform heterogeneous handover. The MIIS server includes neighbor graphs and other related information needed for handover monitoring and decision making.

\section{A. Layer Architecture}

The five layers of the proposed system architecture includes: Application Layer, Transport Layer, Network Layer, Lower Layer and MIHF Layer.

1) Lower Layer: The lower layer has numerous transmission interfaces that can support the mobile requirement in heterogeneous network environment and related information, which are transmitted to the MIHF layer by the SAP interfaces. 
2) Network Layer: This layer adopts IETF-related protocols for various interface communications needed in the heterogeneous network environment. This layer communicates with MIHF layer via the MIH_SAP interface.

3) Transport Layer: The study adopts voice-related protocols in this layer. The SCTP, which is the major channel for voice transmissions, to avoid making use of multi-homing properties not interrupt between wireless network and wired network. In application layer makes use of SIP, adopting UDP that delivers SIP control message and response.

4) Application Layer: The application layer includes the administrator interface, SIP services and Management Module. Administrator interface is referred to as a communication interface between system and user and responds to requests to transmit information. The SIP services send and receive SIP messages. When the system starts, the module sets up an external receiver channel and proceeds to SIP registration and also provides current contact information The study proposes Management Module as Handover Mechanisms, proceeds handover procedure that selects the appropriate network by MIHF layer.

5) MIHF Layer: The study adds an MIHF layer in between Network layer and Lower layer. The MIHF layer provides assists the user during mobility management and handover process. The MIHF layer includes three service modules: MIES, MICS and MIIS.

\section{B. $\quad$ MIH General Architecture}

This proposed IEEE 802.21 architecture defines three service modules to facilitate inter-technology handovers in Media-Independent Handover Services.

The MIES provides lower layers information to upper layers, and upper layers drive handover mechanisms. The IEEE 802.21 protocol sets up numerous events and respondent messages for lower layers, and the upper layers use these messages during handover between two systems. When the lower layers sets up events, it sends related messages to upper layers by network and also provides handover mechanisms. The proposed MIES in this architecture can integrate the original handover mechanisms to enhance system handover efficiency.

The MICS allows effective management and control of different interfaces. During service system handover in the heterogeneous environment, inform user to use parameters and data that proceed to reserve resource, hence user have sufficient handover resources. The study proposes the use of MICS architecture, and the management module can horizontal handover, making use of BSs to ensure speedy handover.

The MIIS provides the framework and corresponding mechanism needed for MIH services to discover available neighboring network information. Other neighbor system related-information, including transmission frequency, system type, and system operator set up and the BS is needed by the user to determine which neighboring network can supply in service ranges, and the BS can then perform network topology acquisition. The proposed IEEE 802.21 protocol can be used by all of the various systems. In the experimental setup, the independent MIIS server provides all MIIS service system in the network environment.

\section{Proposed Management Module}

Figure 4 shows the Management Module for the proposed architecture. The lower MIHF information layers pass through the upper layers. The Management Module divided into three functions: Handover Mechanism, Signal Control and Network Parameters.

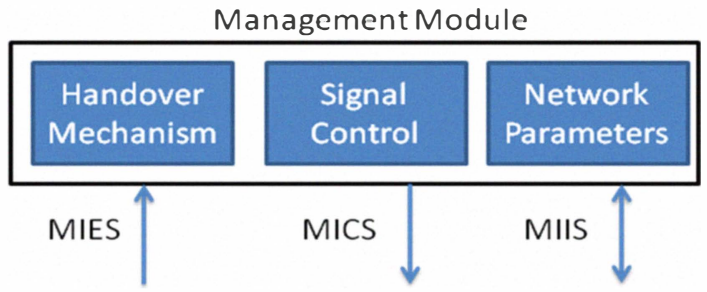

Figure 4. Proposed Management Module

The MIH aims to provide upper layers with the network parameters relevant to the lower layers. Since the terminal can access several network systems simultaneously, the proposed Handover Mechanism selects $f$ networks handover candidates. The study proposes a handover algorithm in the Handover Mechanism module, and Handover Mechanism determines to implement the mechanism that must get the relevant network parameters in Network Parameters module.

The module is responsible for communicating MIHF information. Signal control through MIES enables the Management Module to perform network handover. Handover Mechanism determines Signal Control parameters to receive MIIS information in Network Parameters Module. When the Management Module has passes through the Signal Control to send MICS information. The terminal changes connection from WiMAX system to UMT system. When the terminal transmits WiMAX RSSI ratio lower than the threshold, the MIHF sends 'Link Going Down' signal to Management Module. The Management Module then sends out MICS to MIHF, and MIHF sends nearby environmental parameters to Management Module, and the Management Module passes through the Handover Mechanism selects the next target as handover procedures in UMTS system. When the UMTS set up is complete, the SIP has proceeds to session movement that provides the user for SIP service.

The module stores with management module proceeding before the handover mechanism determine relevant network parameters. The terminal has a network interface that enables mobility in heterogeneous networks. The terminal can therefore cover different network systems simultaneously. The module stores with MIIS terminal and dynamic network information. Users indicate their specific requirements when setting up different QoS network parameters. The study adopts the following parameters: dropping, jitter, bandwidth and RSSI. It provides Handover Mechanism to select appropriate network algorithms. 


\section{Proposed Handover Procedure}

The three parameters considered by the proposed Handover Mechanism are dropping ratio, jitter and bandwidth. Table 1 shows the different network requirements considered in this study, and Table 2 illustrates the four proposed QoS applications, such as interactive class, VoIP-related, Video/Audio streaming and best effort. Application requirements compare with MIIS server that provides to available neighboring network. Interactive class in the first class that request conditions not better in network system, such as web data transmission, so it lowest weight in the table. The second class is VoIP-related that strict request for jitter in voice communication, so candidate network provides jitter lower than application for VoIP request has one weight in the table. The third class is Video/Audio streaming that strict request dropping and bandwidth, providing requirement in candidate network more than application user has one weight in the table. Relatively, in the candidate network get the sum of weight value equal " 2 ". The last class is beset effect that bandwidth request strictly in network, such as FTP file transmission protocol. The application sets the bandwidth request in the table, and the candidate network provides bandwidth conditions get weight value equal " 1 ".

TABLE I. NETWORK PARAMETERS

\begin{tabular}{|l|l|}
\hline D & $\begin{array}{l}\text { Iropping ratio of available netwprk } \\
\text { (From MIIS) }\end{array}$ \\
\hline Dreq $_{\text {request Dropping ratio }}$ & $\begin{array}{l}\text { Requet } \\
\text { (From Application) }\end{array}$ \\
\hline $\mathbf{J}$ & $\begin{array}{l}\text { Jitter of available netwprk } \\
\text { (From MIIS) }\end{array}$ \\
\hline $\mathbf{J}_{\text {reg }}$ & $\begin{array}{l}\text { Request Jitter } \\
\text { (From Application) }\end{array}$ \\
\hline $\mathbf{B}$ & $\begin{array}{l}\text { Bandwidth of available netwprk } \\
\text { (From MIIS) }\end{array}$ \\
\hline $\mathbf{B}_{\text {reg }}$ & $\begin{array}{l}\text { Request Bandwidth } \\
\text { (From Application) }\end{array}$ \\
\hline
\end{tabular}

TABLE II. TRAFFIC TYPES

\begin{tabular}{|c||c|c|c|}
\hline Traffic type & Dropping & Jitter & Bandwidth \\
\hline \hline Class I Interactive class & $\mathrm{O}$ & $\mathrm{O}$ & $\mathrm{O}$ \\
\hline Class2 VolP-related & $\mathrm{O}$ & $\left(\mathrm{J} \leq \mathrm{J}_{\text {reg }}\right) ? 1: 0$ & $O$ \\
\hline Class3 $\begin{array}{c}\text { Video/Audio } \\
\text { streaming }\end{array}$ & $\left.(\mathrm{I}) \leq \mathrm{I})_{\text {reg }}\right) ? 1: 0$ & $O$ & $\left(\mathrm{~B} \leq \mathrm{B}_{\text {reg }}\right) ? 1: 0$ \\
\hline Class4 Best Effort & $\mathrm{O}$ & $\mathrm{O}$ & $\left(\mathrm{B} \leq \mathrm{B}_{\text {reg }}\right) ? 1: 0$ \\
\hline
\end{tabular}

This study hypothesize that the heterogeneous network environment includes UMTS, WiFi and WiMAX networks. The algorithm protocol for selecting from available neighboring networks conforms to handover requirements. The user has soft-handover capability in a heterogeneous network environment. In the example implementation, the user operates telecommunications network equipment, and service providers have numerous $3 \mathrm{G}$ system base stations to optimize mobility in the heterogeneous network environment. Thus, in the proposed handover procedure, the UMTS determines the high-priority networks.

The terminal for providing external communications by UMTS moves continuously among three network interfaces.
Because the BS distance results in continuous RSS decline, the MIHF monitors RSS values lower than RSS threshold values and reports them to the Management Module, which determines handover. To confirm handover, the Management Module requested available network information in MIIS Server by MIHF. The terminals are available networks that communicate with the Management Module, which must calculate available network weights individually before performing the next step.

To determine the type selected for handover in neighboring UMTS system, the steps on the right side of the flow chart are executed sequentially. The Handover Mechanism selects a network from available neighboring networks.

\section{PERFORMANCE ANALYSIS}

This section first introduces the simulation environment and then describes the simulation metrics used to analyze the performance of the proposed Management Module. First, vehicular network performance is simulated with and without Management Module. Then, the overall effect on the media VoIP/Video throughput with and without QoS mechanism is simulated.

\section{A. Simulation Environment}

The performance of proposed Management Module is analyzed by the NS2. The vehicle accesses the services from service providers with VoIP stream and Video stream.

\section{B. Management Module}

The OBU device supports IEEE 802.21 standard in Vehicles. The simulation assumes that four modules, Interface layer, MAC layer, MIHF module, and Handover module have been loaded in nodes.

The simulated network has $3 \mathrm{G}$ and WiMAX with $2 \mathrm{Mbps}$ and $14 \mathrm{Mbps}$ bandwidth capacity, respectively. The UMTS connects to GGSN with $20 \mathrm{Mbps}$ and $802.16 \mathrm{BS}$ connect to CSN with 20Mbps wired link. The SIP-based MIIS server and $\mathrm{CN}$ connect to Internet with $100 \mathrm{Mbps}$. The link capacity between Router and Internet is $8 \mathrm{Mbps}$. Node speed was set to $30 \mathrm{~km} / \mathrm{hr}$. Simulation time was $200 \mathrm{~s}$.

In Fig. 5, the throughput of MIH-based system with supporting vehicle number near 50 nodes is higher than both UMTS and WiMAX in the situation without supporting heterogeneous handover. In Fig. 6, although MIH-based system has a longer average handover time than both UMTS and WiMAX do, sacrificing the tolerated handover time to obtain a significantly higher throughput is (a worthwhile task OR worthwhile). 


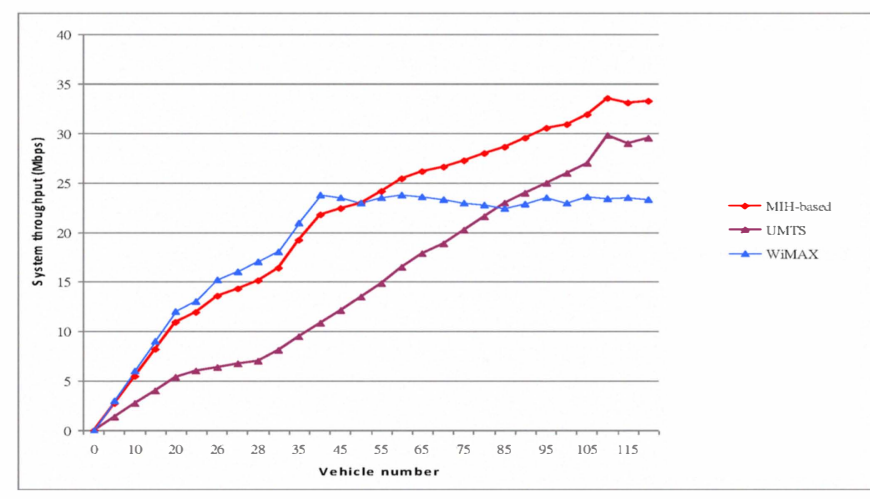

Figure 5. System Throughput

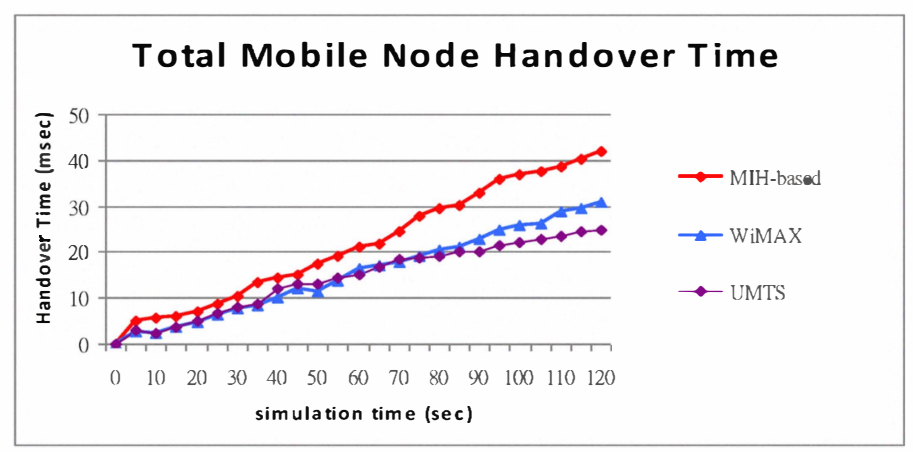

Figure 6. Handover Time

The simulation indicated that, compared to the homogenous network, the proposed MIH Management Module obtained a $46.13 \%$ longer handover time and $27.1 \%$ larger throughput. OR The simulated MIH Management Module obtained a 46.13\% longer handover time and a $27.1 \%$ larger throughput than the homogenous network did.

\section{CONCLUSIONS}

This work designed an IEEE 802.21 MIH mechanism, which was then embedded into OSI layers. This work also designed the management module and adaptive QoS mechanism to improve service performance. The simulation results confirm the effectiveness of the proposed module for vehicular network. The simulation results show that average handover time is slower than both UMTS and WiMAX when the MIH mechanism is used in vehicular network. However, the simulation results confirm that using the IEEE 802.21 MIH mechanism can increase overall throughput. Increased throughput is satisfactory compensation for increased handover time. By the using of Management Module with adaptive QoS mechanism, the proposed module enables users to manage Video/VoIP applications in high-quality network environments.

\section{REFERENCES}

[1] T.Y. Chung, F.C. Yuan, Y.M. Chen, B.J. Liu and C.C. Hsu, "Extending Always Best Connected Paradigm for Voice Communications in Next Generation Wireless Network," Proceedings of the 22nd International Conference on Advanced Information Networking and Applications, pp.803-810, March 2008.

[2] G. Lampropoulos, A.K. Salkintzis and N. Passas, "Media-Independent Handover for Seamless Service Provision in Heterogeneous Networks," IEEE Communications Magazine, Vol.46, No.1, pp.64-71, January 2008.

[3] V.S. Abhayawardhana and R. Babbage, "A Traffic Model for the IP Multimedia Subsystem (IMS)," Proceedings of the IEEE 65th Vehicular Technology Conference, pp.783-787, April 2007.

[4] A. De La Oliva, A. Banchs, I. Soto, T. Melia and A. Vidal, "An Overview of IEEE 802.21: Media-independent Handover Services," IEEE Wireless Communications, Vol.15, No.4, pp.96-103, August 2008.

[5] L. Eastwood, S. Migaldi, Q. Xie and V. Gupta, "Mobility using IEEE 802.21 in a Heterogeneous IEEE 802.16/802.11-based, IMT-advanced (4G) network," IEEE Wireless Communications, Vol.15, No2, pp.26-34, April 2008.

[6] K. Taniuchi, Y. Ohba, V. Fajardo, S. Das, M. Tauil, C. Yuu-Heng, A. Dutta, D. Baker, M. Yajnik and D. Famolari, "IEEE 80221: Media Independent Handover: Features, Applicability, and Realization," IEEE Communications Magazine, Vol.47, No.1, pp.112-120, January 2009.

[7] W. Ying, Z. Yun, Y. Jun and Z. Ping, "An Enhanced Media Independent Handover Framework for Heterogeneous Networks," Proceedings of the IEEE Vehicular Technology Conference, pp.23062310, May 2008.

[8] G.P. Silvana and H. Schulzrinne, "SIP and 802.21 for Service Mobility and Pro-active Authentication," Proceedings of the Communication Networks and Services Research Conference, pp.176-182, May 2008.

[9] M.I. Corici, A.F. Murarasu, S. Arbanowski, T. Magedanz, S. Lee and X. Liu, "Multimedia Mobility Service Solution," Proceedings of the IEEE Vehicular Technology Conference, pp.1-5, September 2008. 